PROCEEDINGS OF THE

AMERICAN MATHEMATICAL SOCIETY

Volume 133, Number 8, Pages 2219-2224

S 0002-9939(05)07654-9

Article electronically published on March 14, 2005

\title{
IRREDUCIBILITY OF THE (-1)-CLASSES ON SMOOTH RATIONAL SURFACES
}

\author{
MUSTAPHA LAHYANE \\ (Communicated by Michael Stillman) \\ This research is dedicated to my mother, Hnia Hamami
}

\begin{abstract}
We give a characterization for a $(-1)$-divisor $D$ on a smooth rational surface $X$ to be irreducible under the assumption that an anticanonical divisor $-K_{X}$ of $X$ is nef. Here $-K_{X}$ is nef means $K_{X} \cdot C \leq 0$ for every effective divisor $C$ on $X$, and a $(-1)$-divisor $D$ is a divisor such that the two numerical conditions $D^{2}=-1=D \cdot K_{X}$ hold.

As an application we give explicit examples of blowing up the projective plane at nine points infinitely near such that the obtained surface has an infinite number of $(-1)$-curves. A (-1)-curve is a smooth rational curve of self-intersection -1 .
\end{abstract}

\section{INTRODUCTION}

Given a smooth rational surface $X$ for which an anticanonical divisor $-K_{X}$ is nef, we would like to know if a divisor $E$ on $X$ satisfying the two numerical conditions $E^{2}=-1$ and $E \cdot K_{X}=-1$ ( $E$ will be called a $(-1)$-divisor on $\left.X\right)$ is irreducible (hence reduced) or not. Such a divisor $E$ is always effective, that is, the group of sections of the invertible sheaf $\mathcal{O}_{X}(E)$ associated to $E$ has a nontrivial section (see Lemma 2.2 below). Unfortunately, it may be neither reduced nor irreducible. One of our motivations to consider such a question is to have a criterion to check if $X$ has an infinite number of $(-1)$-curves or not. For example, when the self-intersection of an anticanonical divisor of $X$ is zero, it is not difficult to find an infinite family of divisors on $X$ such that each member $E$ of the family is a (-1)-divisor.

Nagata ([6]) proved that each (-1)-divisor on the surface obtained by blowing up 9 points in general position of the projective plane is irreducible, and then it is a $(-1)$-curve, that is, a smooth rational curve of self-intersection -1 . We are interested in giving a criterion to check whether a (-1)-divisor $E$ on $X$ is irreducible reduced or not.

The plan of this note is as follows: in section 2, we give some standard facts about smooth rational surfaces and fix our notation. Section 3 contains our main

Received by the editors June 28, 2001 and, in revised form, October 15, 2003.

2000 Mathematics Subject Classification. Primary 14J26; Secondary 14F05.

Key words and phrases. Smooth rational surfaces, anticanonical divisor, Hodge Index Theorem, points in general position, Néron-Severi group, blowing-up.

The author was partially supported by a postdoctoral fellowship at the International Centre for Theoretical Physics (Trieste, Italy) and a Marie Curie grant number HPMD-GH-01-00097-01 at the department of "Álgebra, Geometría y Topología" of Valladolid University (Valladolid, Spain).

(c) 2005 American Mathematical Society Reverts to public domain 28 years from publication 
result, which gives a necessary and sufficient condition for a (-1)-divisor to be reduced and irreducible. In section 4, there are some applications: the surface obtained by blowing up 9 infinitely near points of the projective plane such that the configuration of the set of $(-2)$-curves is of type $A_{8}$ (respectively $D_{8}, E_{8}$ and $\left.A_{1} A_{7}\right)$ has an infinite number of $(-1)$-curves. A $(-2)$-curve is a smooth rational curve of self-intersection -2 .

\section{Preliminaries}

From now on $X$ will be a smooth rational surface such that $-K_{X}$ is nef, where $K_{X}$ is a canonical divisor on $X$. Here the condition $-K_{X}$ is nef means that $K_{X} . C \leq$ 0 for every effective divisor $C$ on $X$, where . denotes the intersection form on the group of divisors on $X$.

In this context, the integer $K_{X}^{2}$ is greater than or equal to zero (see e.g. [3] Proposition 4, page 729]). Also, it is easy to see that the self-intersection of every reduced irreducible curve on $X$ is greater than or equal to -2 . In particular, define a $(-2)$-curve on $X$ as a smooth rational curve of self-intersection -2 and a (-1)-curve as one with self-intersection -1 .

Definition 2.1. Let $E$ be a divisor on $X . E$ is a (-1)-divisor if and only if $E^{2}=-1=E . K_{X}$. The class of a $(-1)$-divisor in the Néron-Severi group of $X$ will be called a $(-1)$-class, or simply a $(-1)$-divisor.

The following lemma shows that a (-1)-divisor on $X$ is always effective.

Lemma 2.2. Every (-1)-divisor on $X$ is effective.

Proof. Let $E$ be a (-1)-divisor on $X$. The Riemann-Roch theorem applied to the invertible sheaf $\mathcal{O}_{X}(E)$ gives the following inequality:

$$
h^{0}\left(X, \mathcal{O}_{X}(E)\right)+h^{0}\left(X, \mathcal{O}_{X}\left(K_{X}-E\right)\right) \geq 1 .
$$

Since $K_{X} \cdot\left(K_{X}-E\right)=K_{X}^{2}+1>0$, the divisor $K_{X}-E$ is not effective. So we are done.

Remark 2.3. As the proof suggests, the result of Lemma 2.2 holds for any smooth rational surface having an anticanonical divisor of self-intersection greater than or equal to zero.

Remark 2.4. In [2], there are examples which prove that a (-1)-divisor may not be effective if $K_{X}^{2}<0$.

We will need the following well-known result (see for instance [4]):

Lemma 2.5. Let $X$ be a smooth projective rational surface such that the selfintersection of its canonical divisor $K_{X}$ is zero. Then the intersection form is negative semi-definite on $K_{X}^{\perp}$; and for every element $x \in K_{X}^{\perp}$, the following equivalence holds:

$$
x^{2}=0 \text { if and only if } q x=p K_{X} \text { for some integers } p \text { and } q \neq 0 .
$$

$K_{X}^{\perp}$ is the set of all divisors $D$ on $X$ which satisfy D. $K_{X}=0$.

We give here an elementary proof. Let $x$ be an element of $K_{X}^{\perp}$. We can suppose that $x$ is the divisor class of a divisor $D$ on $X$. If $D^{2}$ is larger than zero, then 
by using the Hodge Index Theorem (see e.g. [5], [1]), $K_{X}$ would be either of selfintersection less than zero or numerically trivial. These are impossible since $X$ is rational and $K_{X}^{2}=0$.

Let $A$ be an ample divisor on $X$, and assume furthermore that $D^{2}=0$. Consider the divisor $E$ defined as $E=\left(A . K_{X}\right) D-(A . D) K_{X}$. We have $E^{2}=0=A$. $E$. Hence, by the Hodge Index Theorem, the divisor $\left(A . K_{X}\right) D$ is numerically equivalent to $(A . D) K_{X}$. Since $X$ is rational and $-K_{X}$ is an effective divisor, we obtain that

$$
D=\frac{A \cdot D}{A \cdot K_{X}} K_{X}
$$

\section{THE CRITERION}

Let $\left\{C_{1}, \ldots, C_{r}\right\}$ be the set of $(-2)$-curves on $X$ ( $r$ is a nonnegative integer which can be zero if $X$ has no (-2)-curves). The following is our main result.

Theorem 3.1. Let $X$ be a smooth rational surface having an anticanonical divisor being nef and let $\left\{C_{1}, \ldots, C_{r}\right\}$ be the set of $(-2)$-curves on $X$, where $r$ is a nonnegative integer. Then, the following assertions are equivalent for a (-1)-divisor $E$ on $X$ :

1. E is a (-1)-curve.

2. E. $C_{i} \geq 0$ for every $i=1, \ldots, r$.

Proof. It is obvious that if $E$ is a (-1)-curve on $X$, then the intersection number of $E$ with any (-2)-curve on $X$ is greater than or equal to zero.

Conversely, let $E$ be a (-1)-divisor such that the numerical conditions E. $C_{i} \geq 0$ for every $i=1, \ldots, r$ hold. By Lemma 2.2, $E$ is effective; hence $E$ is linearly equivalent to $E_{0}+\sum_{i=1}^{i=r} b_{i} C_{i}$, where $E_{0}$ is an effective divisor having no (-2)-curve as a component, and the $b_{i}$ are nonnegative integers for every $i=1, \ldots, r$. The condition $E^{2}=-1$ gives the following equality:

$$
-1=E_{0}^{2}+\sum_{i=1}^{i=r} b_{i}\left(E_{0} \cdot C_{i}+E . C_{i}\right)
$$

Hence, $E_{0}^{2} \leq-1$ (since $C_{i} . E_{0} \geq 0$ and $C_{i} . E \geq 0$ for all $\left.i=1, \ldots, r\right)$.

The condition $E$. $\left(-K_{X}\right)=1$ implies the equality: $E_{0} \cdot\left(-K_{X}\right)=1$. If $\Gamma_{0}, \Gamma_{1}, \ldots$, $\Gamma_{\sigma}$ are the components of the effective divisor $E_{0}$, i.e., $E_{0}=\sum_{i=0}^{i=\sigma} a_{i} \Gamma_{i}$, then

$$
1=a_{0} \Gamma_{0} \cdot\left(-K_{X}\right)+\ldots+a_{\sigma} \Gamma_{\sigma} \cdot\left(-K_{X}\right) .
$$

Since the divisor $\left(-K_{X}\right)$ is nef, we can suppose that $a_{0} \Gamma_{0} \cdot\left(-K_{X}\right)=1$; so we have $a_{0}=\Gamma_{0} \cdot\left(-K_{X}\right)=1$ and $a_{i} \Gamma_{i} \cdot\left(-K_{X}\right)=0$ for every $i=1, \ldots, \sigma$.

We distinguish between two cases:

Case where $K_{X}^{2}>0$ : Let $i$ be any element of the set $\{1,2, \ldots, \sigma\}$. From $a_{i} \Gamma_{i} \cdot\left(-K_{X}\right)=0$, we will prove that $a_{i}=0$. To do so, if $\Gamma_{i} \cdot\left(-K_{X}\right)=0$, then by the Hodge Index Theorem the only case which can occur is $\Gamma_{i}^{2}<0$, that is, by the adjunction formula $\Gamma_{i}$ is a $(-2)$-curve which is impossible. Hence $a_{i}=0$. In conclusion, $E_{0}=\Gamma_{0}$. But $-1 \leq \Gamma_{0}^{2}$ and $E_{0}^{2} \leq-1$; hence $E_{0}$ is a $(-1)$-curve.

Case where $K_{X}^{2}=0$ : In this case for every $i=1, \ldots, \sigma$, we have $-2 \leq \Gamma_{i}^{2} \leq$ 0 . The only case which can occur is $\Gamma_{i}^{2} \geq 0$. Then there exist some natural integers $p_{i}$ and $q_{i}, i=1, \ldots, \sigma\left(q_{i} \neq 0\right.$ for all $\left.i=1, \ldots, \sigma\right)$, such that $q_{i} \Gamma_{i}=p_{i}\left(-K_{X}\right)$ for all $i=1, \ldots, \sigma$. Consequently, there exist two natural 
integers $N\left(N=q_{1} \ldots q_{\sigma}\right)$, and $a$ such that: $N E_{0}=N \Gamma_{0}+a\left(-K_{X}\right)$. Hence by using the adjunction formula applied to $\Gamma_{0}$, we obtain that $\Gamma_{0}$ is a $(-1)$ curve and $a=0$. In other words, $E_{0}$ is a $(-1)$-curve on $X$.

So in both cases, $E_{0}$ is a $(-1)$-curve. Hence we have the equalities:

$$
\text { E. } \sum_{i=1}^{i=r} b_{i} C_{i}=E_{0} \cdot \sum_{i=1}^{i=r} b_{i} C_{i}=0 .
$$

Now define $C$ as $C=\sum_{i=1}^{i=r} b_{i} C_{i}$. Then $C^{2}=0$. It follows immediately that there exist some natural integers $p$ and $q(q \neq 0)$ such that $q C=p\left(-K_{X}\right)$; hence $q E=q E_{0}+p\left(-K_{X}\right)$.

The facts that $E$ is a $(-1)$-divisor, $E_{0}$ is a $(-1)$-curve and $q \neq 0$ prove that the complete linear system $|E|$ associated to $E$ is equal to the singleton $\left\{E_{0}\right\}$ having the (-1)-curve $E_{0}$ as its unique element.

\section{Some APplicAtions}

In this section, we apply our criterion to prove that certain blowings-up of the projective plane at nine points have an infinite number of $(-1)$-curves. The method consists in giving an explicit infinite family of $(-1)$-curves.

Since $X$ is a blow-up of the projective plane at nine points (may be infinitely near), the Néron-Severi group $N S(X)$ of $X$ has a basis $\left(\mathcal{E}_{0}, \mathcal{E}_{1}, \ldots, \mathcal{E}_{9}\right)$ defined by:

- $\mathcal{E}_{0}$ is the class of lines on the projective plane which do not pass through any of the nine points in consideration;

- $\mathcal{E}_{i}$ is the class of the exceptional divisor corresponding to the $i$ th point being blown up for every $i=1, \ldots, 9$.

The class of a divisor on $X$ will be represented by the 10-tuple $\left(d, m_{1}, \ldots, m_{9}\right)$; in particular, if $m_{i}=m_{i+1}=\ldots=m_{j}=m, 1 \leq i<j \leq 9$, we write this class as $\left(d, m_{1}, \ldots, m_{i-1}, m^{j-i+1}, m_{j+1}, \ldots, m_{9}\right)$.

The intersection form on $N S(X)$ is given by:

- $\mathcal{E}_{0}^{2}=1=-\mathcal{E}_{i}^{2}$ for every $i=1, \ldots, 9$;

- $\mathcal{E}_{i} \cdot \mathcal{E}_{j}=0$ for every $i, j=0,1, \ldots, 9$, with $i \neq j$.

Example 4.1. Taking the first point in the projective plane, the second point will be a general point on the exceptional divisor, the third point will be a general point on the exceptional divisor of the blowing-up of the second point, and so on. Then the classes of $(-2)$-curves are : $\left(0,-1,1,0^{7}\right),\left(0,0,-1,1,0^{6}\right),\left(0,0^{2},-1,1,0^{5}\right)$, $\left(0,0^{3},-1,1,0^{4}\right),\left(0,0^{4},-1,1,0^{3}\right),\left(0,0^{5},-1,1,0^{2}\right),\left(0,0^{6},-1,1,0\right),\left(0,0^{7},-1,1\right)$.

For each positive integer $d$, the class $\left(3 d^{2},\left(d^{2}+d\right),\left(d^{2}\right)^{6},\left(d^{2}-1\right),\left(d^{2}-d\right)\right)$ is the class of a (-1)-curve on $X$ (hence $X$ has an infinite number of $(-1)$-curves). Indeed, we have:

- $\left(3 d^{2},\left(d^{2}+d\right),\left(d^{2}\right)^{6},\left(d^{2}-1\right),\left(d^{2}-d\right)\right) \cdot\left(0,-1,1,0^{7}\right)=d$,

- the intersection number of $\left(3 d^{2},\left(d^{2}+d\right),\left(d^{2}\right)^{6},\left(d^{2}-1\right),\left(d^{2}-d\right)\right)$ with any $\left(0,0,-1,1,0^{6}\right),\left(0,0^{2},-1,1,0^{5}\right),\left(0,0^{3},-1,1,0^{4}\right),\left(0,0^{4},-1,1,0^{3}\right),\left(0,0^{5}\right.$, $\left.-1,1,0^{2}\right)$ is equal to zero,

- $\left(3 d^{2},\left(d^{2}+d\right),\left(d^{2}\right)^{6},\left(d^{2}-1\right),\left(d^{2}-d\right)\right) \cdot\left(0,0^{6},-1,1,0\right)=1$,

- $\left(3 d^{2},\left(d^{2}+d\right),\left(d^{2}\right)^{6},\left(d^{2}-1\right),\left(d^{2}-d\right)\right) \cdot\left(0,0^{7},-1,1\right)=d-1$. 
Example 4.2. Taking the first point in the projective plane, the second point will be a general point on the exceptional divisor, the third point will be a general point on the exceptional divisor of the second point blown-up, and so on until the eighth point. The ninth point will be an ordinary point in the projective plane such that it belongs to the line determined by the first point and the second point (which is an infinitely near point of the first point). Then the classes of $(-2)$-curves are: $\left(0,-1,1,0^{7}\right),\left(0,0,-1,1,0^{6}\right),\left(0,0^{2},-1,1,0^{5}\right),\left(0,0^{3},-1,1,0^{4}\right)$, $\left(0,0^{4},-1,1,0^{3}\right),\left(0,0^{5},-1,1,0^{2}\right),\left(0,0^{6},-1,1,0\right),\left(1,1,1,0^{6}, 1\right)$.

For integers $p \geq 0$ and $q \geq 0$, the class $\left(2^{p} \times 3^{q+1} \times\left(2^{p} \times 3^{q}-1\right),\left(4^{p} \times 9^{q}-1\right)\right.$, $\left.\left(2^{p} \times 3^{q} \times\left(2^{p} \times 3^{q}-1\right)\right)^{7},\left(2^{p+1} \times 3^{q} \times\left(2^{p-1} \times 3^{q}-1\right)\right)\right)$ is the class of a $(-1)$-curve on $X$ (hence $X$ has an infinite number of $(-1)$-curves). Indeed, we have:

- $\left(2^{p} \times 3^{q+1} \times\left(2^{p} \times 3^{q}-1\right),\left(4^{p} \times 9^{q}-1\right),\left(2^{p} \times 3^{q} \times\left(2^{p} \times 3^{q}-1\right)\right)^{7}\right.$, $\left.\left(2^{p+1} \times 3^{q} \times\left(2^{p-1} \times 3^{q}-1\right)\right)\right) \cdot\left(0,-1,1,0^{7}\right)=\left(2^{p} \times 3^{q}-1\right)$,

- the intersection number of $\left(2^{p} \times 3^{q+1} \times\left(2^{p} \times 3^{q}-1\right),\left(4^{p} \times 9^{q}-1\right)\right.$, $\left.\left(2^{p} \times 3^{q} \times\left(2^{p} \times 3^{q}-1\right)\right)^{7},\left(2^{p+1} \times 3^{q} \times\left(2^{p-1} \times 3^{q}-1\right)\right)\right)$ with any $\left(0,0,-1,1,0^{6}\right)$, $\left(0,0^{2},-1,1,0^{5}\right),\left(0,0^{3},-1,1,0^{4}\right),\left(0,0^{4},-1,1,0^{3}\right),\left(0,0^{5},-1,1,0^{2}\right),\left(0,0^{6}\right.$, $-1,1,0)$ is equal to zero.

- $\left(2^{p} \times 3^{q+1} \times\left(2^{p} \times 3^{q}-1\right),\left(4^{p} \times 9^{q}-1\right),\left(2^{p} \times 3^{q} \times\left(2^{p} \times 3^{q}-1\right)\right)^{7}\right.$, $\left.\left(2^{p+1} \times 3^{q} \times\left(2^{p-1} \times 3^{q}-1\right)\right)\right) \cdot\left(1,1,1,0^{6}, 1\right)=1$.

Example 4.3. Taking the first point in the projective plane, the second point will be a general point on the exceptional divisor, the third point will be the point belonging to the exceptional divisor of the blowing-up of the second point and the strict transform of the line determined by the first ordinary point and the second point. The fourth point will be a general point on the third exceptional divisor and so on until the eighth point. The ninth point will be an ordinary point such that it does not belong to the line determined by the first point and the second point (which is an infinitely near point of the first point). Then the classes of $(-2)$-curves are: $\left(0,-1,1,0^{7}\right),\left(0,0,-1,1,0^{6}\right),\left(0,0^{2},-1,1,0^{5}\right),\left(0,0^{3},-1,1,0^{4}\right)$, $\left.\left(0,0^{4},-1,1,0^{3}\right),\left(0,0^{5},-1,1,0^{2}\right),\left(0,0^{6},-1,1,0\right),\left(1,1,1,1,0^{6}\right)\right)$.

For each integer $p$, the class $\left(6 \times\left(p^{2}+1\right) \times\left(2 \times p^{2}+1\right),\left(2 \times\left(p^{2}+1\right) \times\left(2 \times p^{2}+1\right)\right)^{7}\right.$, $\left.4 \times p^{2} \times\left(p^{2}+1\right), 4 \times p^{2} \times\left(p^{2}+2\right)+3\right)$ is the class of a $(-1)$-curve on $X$ (hence $X$ has an infinite number of (-1)-curves). Indeed, we have:

- The intersection number of $\left(6 \times\left(p^{2}+1\right) \times\left(2 \times p^{2}+1\right),\left(2 \times\left(p^{2}+1\right) \times\right.\right.$ $\left.\left.\left(2 \times p^{2}+1\right)\right)^{7}, 4 \times p^{2} \times\left(p^{2}+1\right), 4 \times p^{2} \times\left(p^{2}+2\right)+3\right)$ with any $\left(0,-1,1,0^{7}\right)$, $\left(0,0,-1,1,0^{6}\right),\left(0,0^{2},-1,1,0^{5}\right),\left(0,0^{3},-1,1,0^{4}\right),\left(0,0^{4},-1,1,0^{3}\right),\left(0,0^{5}\right.$, $\left.\left.-1,1,0^{2}\right),\left(1,1,1,1,0^{6}\right)\right)$ is equal to zero,

- $\left(6 \times\left(p^{2}+1\right) \times\left(2 \times p^{2}+1\right),\left(2 \times\left(p^{2}+1\right) \times\left(2 \times p^{2}+1\right)\right)^{7}, 4 \times p^{2} \times\right.$ $\left.\left(p^{2}+1\right), 4 \times p^{2} \times\left(p^{2}+2\right)+3\right) \cdot\left(0,0^{6},-1,1,0\right)=2 \times\left(p^{2}+1\right)$.

Example 4.4. Taking the first point in the projective plane, the second point will be a general point on the exceptional divisor, and so on until the seventh point. The eighth point will be an ordinary point, and the ninth point will be an infinitely near point of the eighth point and will belong to the line determined by the first point and the eighth point. Then the classes of $(-2)$-curves are: $\left(0,-1,1,0^{7}\right)$, $\left(0,0,-1,1,0^{6}\right),\left(0,0^{2},-1,1,0^{5}\right),\left(0,0^{3},-1,1,0^{4}\right),\left(0,0^{4},-1,1,0^{3}\right),\left(0,0^{5},-1,1,0^{2}\right)$, $\left(0,0^{7},-1,1\right)$ and $\left.\left(1,1,0^{6}, 1,1\right)\right)$. 
The class $\left(3^{d+1} \times\left(3^{d}+1\right), 3^{d} \times\left(3^{d}+2\right),\left(3^{d} \times\left(3^{d}+1\right)\right)^{7}, 3^{2 d}-1\right)$ is irreducible for each positive integer $d$ (hence $X$ has an infinite number of (-1)-curves). Indeed, we have:

- $\left(3^{d+1} \times\left(3^{d}+1\right), 3^{d} \times\left(3^{d}+2\right),\left(3^{d} \times\left(3^{d}+1\right)\right)^{7}, 3^{2 d}-1\right) \cdot\left(0,-1,1,0^{7}\right)=3^{d}$,

- the intersection number of

$$
\left(3^{d+1} \times\left(3^{d}+1\right), 3^{d} \times\left(3^{d}+2\right),\left(3^{d} \times\left(3^{d}+1\right)\right)^{7}, 3^{2 d}-1\right)
$$

with any $\left(0,0,-1,1,0^{6}\right),\left(0,0^{2},-1,1,0^{5}\right),\left(0,0^{3},-1,1,0^{4}\right),\left(0,0^{4},-1,1,0^{3}\right)$, $\left(0,0^{5},-1,1,0^{2}\right)$ is equal to zero.

- $\left(3^{d+1} \times\left(3^{d}+1\right), 3^{d} \times\left(3^{d}+2\right),\left(3^{d} \times\left(3^{d}+1\right)\right)^{7}, 3^{2 d}-1\right) \cdot\left(0,0^{7},-1,1\right)=3^{d}+1$,

- $\left.\left(3^{d+1} \times\left(3^{d}+1\right), 3^{d} \times\left(3^{d}+2\right),\left(3^{d} \times\left(3^{d}+1\right)\right)^{7}, 3^{2 d}-1\right) \cdot\left(1,1,0^{6}, 1,1\right)\right)=1$.

\section{ACKNOWLEDGEMENTS}

Part of this work was done at Laboratoire J. A. Dieudonné, U.M.R. C.N.R.S. No. 6621, Université de Nice Sophia-Antipolis (Nice, France). Many thanks to Professors Gang Xiao and André Hirschowitz. I would like also to acknowledge the hospitality of the Abdus Salam International Centre for Theoretical Physics (Trieste, Italy), without which this work would not have been possible. The revised version was done at the Department of "Álgebra, Geometría y Topología" of the Valladolid University where there exists a very nice atmosphere to work. This research is dedicated to my mother, Hnia Hamami, who lost her life on June 28, 2003. Life, whatever its form, is expensive and should be protected.

\section{REFERENCES}

1. W. Barth, C. Peters, A. Van de Ven, Compact Complex Surfaces. Berlin, Springer (1984). MR0749574 (86c:32026)

2. B. Harbourne, Private communication.

3. B. Harbourne, Rational surfaces with $K^{2}>0$, Proceedings of the American Mathematical Society, Volume 124, Number 3, March 1996. MR1307526 (96f:14045)

4. B. Harbourne, Anticanonical rational surfaces, Transactions of the American Mathematical Society, Volume 349 (1997), Number 3, 1191-1208. MR1373636 (97f:14007)

5. R. Hartshorne, Algebraic Geometry, Graduate Texts in Mathematics, Springer Verlag (1977). MR0463157 (57:3116)

6. M. Nagata, On rational surfaces, II, Mem. Coll. Sci. Univ. Kyoto, Ser. A Math. 33 (1960), 271-293. MR0126444 (23:A3740)

Abdus Salam International Centre for Theoretical Physics, 34100 Trieste, Italy Current address: Departamento de Àlgebra, Geometrìa y Topologìa, Facultad de Ciencias, 47005 Valladolid, Spain

E-mail address: lahyane@agt.uva.es 